Pacific

Journal of

Mathematics

ON THE VERE-JONES CLASSIFICATION AND

EXISTENCE OF MAXIMAL MEASURES FOR COUNTABLE TOPOLOGICAL MARKOV CHAINS

Sylvie Ruette 


\title{
ON THE VERE-JONES CLASSIFICATION AND EXISTENCE OF MAXIMAL MEASURES FOR COUNTABLE TOPOLOGICAL MARKOV CHAINS
}

\author{
Sylvie Ruette
}

\begin{abstract}
We consider topological Markov chains (also called Markov shifts) on countable graphs. We show that a transient graph can be extended to a recurrent graph of equal entropy which is either positive recurrent of null recurrent, and we give an example of each type. We extend the notion of local entropy to topological Markov chains and prove that a transitive Markov chain admits a measure of maximal entropy (or maximal measure) whenever its local entropy is less than its (global) entropy.
\end{abstract}

\section{Introduction.}

In this article we are interested in connected oriented graphs and topological Markov chains. All the graphs we consider have a countable set of vertices. If $G$ is an oriented graph, let $\Gamma_{G}$ be the set of two-sided infinite sequences of vertices that form a path in $G$ and let $\sigma$ denote the shift transformation. The Markov chain associated to $G$ is the (noncompact) dynamical system $\left(\Gamma_{G}, \sigma\right)$. The entropy $h(G)$ of the Markov chain $\Gamma_{G}$ was defined by Gurevich; it can be computed by several ways and satisfies the Variational Principle $[7]$ and $[8]$.

In [16] Vere-Jones classifies connected oriented graphs as transient, null recurrent or positive recurrent according to the properties of the series associated with the number of loops, by analogy with probabilistic Markov chains. To a certain extent, positive recurrent graphs resemble finite graphs. In [7] Gurevich shows that a Markov chain on a connected graph admits a measure of maximal entropy (also called maximal measure) if and only if the graph is positive recurrent. In this case, this measure is unique and it is an ergodic Markov measure.

In [13] and [14] Salama gives a geometric approach to the Vere-Jones classification. The fact that a graph can (or cannot) be "extended" or "contracted" without changing its entropy is closely related to its class. In particular a graph with no proper subgraph of equal entropy is positive recurrent. The converse is not true [14] (see also [6] for an example of a positive recurrent graph with a finite valency at every vertex that has 
no proper subgraph of equal entropy). This result shows that the positive recurrent class splits into two subclasses: A graph is called strongly positive recurrent if it has no proper subgraph of equal entropy; it is equivalent to a combinatorial condition (a finite connected graph is always strongly positive recurrent). In [13] and [14] Salama also states that a graph is transient if and only if it can be extended to a bigger transient graph of equal entropy. We show that any transient graph $G$ is contained in a recurrent graph of equal entropy, which is positive or null recurrent depending on the properties of $G$. We illustrate the two possibilities - a transient graph with a positive or null recurrent extension - by an example.

The result of Gurevich entirely solves the question of existence of a maximal measure in term of graph classification. Nevertheless it is not so easy to prove that a graph is positive recurrent and one may wish to have more efficient criteria. In [10] Gurevich and Zargaryan give a sufficient condition for existence of a maximal measure; it is formulated in terms of exponential growth of the number of paths inside and outside a finite subgraph. We give a new sufficient criterion based on local entropy.

Why consider local entropy? For a compact dynamical system, it is known that a null local entropy implies the existence of a maximal measure ([11], see also [1] for a similar but different result). This result may be strengthened in some cases: It is conjectured that, if $f$ is a map of the interval which is $C^{r}$, $r>1$, and satisfies $h_{\text {top }}(f)>h_{\text {loc }}(f)$, then there exists a maximal measure [2]. Our initial motivation comes from the conjecture above because smooth interval maps and Markov chains are closely related. If $f:[0,1] \rightarrow[0,1]$ is $C^{1+\alpha}$ (i.e., $f$ is $C^{1}$ and $f^{\prime}$ is $\alpha$-Hölder with $\alpha>0$ ) with $h_{\text {top }}(f)>0$ then an oriented graph $G$ can be associated to $f, G$ is connected if $f$ is transitive, and there is a bijection between the maximal measures of $f$ and those of $\Gamma_{G}[\mathbf{2}]$ and [3]. We show that a Markov chain is strongly positive recurrent, thus admits a maximal measure, if its local entropy is strictly less that its Gurevich entropy. However this result does not apply directly to interval maps since the "isomorphism" between $f$ and its Markov extension is not continuous so it may not preserve local entropy (which depends on the distance).

The article is organized as follows. Section 1 contains definitions and basic properties on oriented graphs and Markov chains. In Section 2, after recalling the definitions of transient, null recurrent and positive recurrent graphs and some related properties, we show that any transient graph is contained in a recurrent graph of equal entropy (Proposition 2.8) and we give an example of a transient graph which extends to a positive recurrent (resp. null recurrent) graph. Section 3 is devoted to the problem of existence of maximal measures: Theorem 3.8 gives a sufficient condition for the existence of a maximal measure, based on local entropy. 


\section{Background.}

1.1. Graphs and paths. Let $G$ be an oriented graph with a countable set of vertices $V(G)$. If $u, v$ are two vertices, there is at most one arrow $u \rightarrow v$. A path of length $n$ is a sequence of vertices $\left(u_{0}, \ldots, u_{n}\right)$ such that $u_{i} \rightarrow u_{i+1}$ in $G$ for $0 \leq i<n$. This path is called a loop if $u_{0}=u_{n}$. We say that the graph $G$ is connected if for all vertices $u, v$ there exists a path from $u$ to $v$; in the literature, such a graph is also called strongly connected.

If $H$ is a subgraph of $G$, we write $H \subset G$; if in addition $H \neq G$, we write $H \varsubsetneqq G$ and say that $H$ is a proper subgraph. If $W$ is a subset of $V(G)$, the set $V(G) \backslash W$ is denoted by $\bar{W}$. We also denote by $W$ the subgraph of $G$ whose vertices are $W$ and whose edges are all edges of $G$ between two vertices in $W$.

Let $u, v$ be two vertices. We define the following quantities:

- $p_{u v}^{G}(n)$ is the number of paths $\left(u_{0}, \ldots, u_{n}\right)$ such that $u_{0}=u$ and $u_{n}=v$; $R_{u v}(G)$ is the radius of convergence of the series $\sum p_{u v}^{G}(n) z^{n}$.

- $f_{u v}^{G}(n)$ is the number of paths $\left(u_{0}, \ldots, u_{n}\right)$ such that $u_{0}=u, u_{n}=v$ and $u_{i} \neq v$ for $0<i<n ; L_{u v}(G)$ is the radius of convergence of the series $\sum f_{u v}^{G}(n) z^{n}$.

Proposition 1.1 (Vere-Jones [16]). Let $G$ be an oriented graph. If $G$ is connected, $R_{u v}(G)$ does not depend on $u$ and $v$; it is denoted by $R(G)$.

If there is no confusion, $R(G)$ and $L_{u v}(G)$ will be written $R$ and $L_{u v}$. For a graph $G^{\prime}$ these two radii will be written $R^{\prime}$ and $L_{u v}^{\prime}$.

1.2. Markov chains. Let $G$ be an oriented graph. $\Gamma_{G}$ is the set of twosided infinite paths in $G$, that is,

$$
\Gamma_{G}=\left\{\left(v_{n}\right)_{n \in \mathbb{Z}} \mid \forall n \in \mathbb{Z}, v_{n} \rightarrow v_{n+1} \text { in } G\right\} \subset(V(G))^{\mathbb{Z}} .
$$

$\sigma$ is the shift on $\Gamma_{G}$. The (topological) Markov chain on the graph $G$ is the system $\left(\Gamma_{G}, \sigma\right)$.

The set $V(G)$ is endowed with the discrete topology and $\Gamma_{G}$ is endowed with the induced topology of $(V(G))^{\mathbb{Z}}$. The space $\Gamma_{G}$ is not compact unless $G$ is finite. A compatible distance on $\Gamma_{G}$ is given by $d$, defined as follows: $V(G)$ is identified with $\mathbb{N}$ and the distance $D$ on $V(G)$ is given by $D(n, m)=$ $\left|\frac{1}{2^{n}}-\frac{1}{2^{m}}\right|$. If $\bar{u}=\left(u_{n}\right)_{n \in \mathbb{Z}}$ and $\bar{v}=\left(v_{n}\right)_{n \in \mathbb{Z}}$ are two elements of $\Gamma_{G}$,

$$
d(\bar{u}, \bar{v})=\sum_{n \in \mathbb{Z}} \frac{D\left(u_{n}, v_{n}\right)}{2^{|n|}} \leq 3 .
$$

The Markov chain $\left(\Gamma_{G}, \sigma\right)$ is transitive if for any nonempty open sets $A, B \subset \Gamma_{G}$ there exists $n>0$ such that $\sigma^{n}(A) \cap B \neq \emptyset$. Equivalently, $\Gamma_{G}$ is transitive if and only if the graph $G$ is connected. In the sequel we will be interested in connected graphs only. 
1.3. Entropy. If $G$ is a finite graph, $\Gamma_{G}$ is compact and the topological entropy $h_{\text {top }}\left(\Gamma_{G}, \sigma\right)$ is well-defined (see e.g., [5] for the definition of the topological entropy). If $G$ is a countable graph, the Gurevich entropy [7] of $G$ is given by

$$
h(G)=\sup \left\{h_{\text {top }}\left(\Gamma_{H}, \sigma\right) \mid H \subset G, H \text { finite }\right\} .
$$

This entropy can also be computed in a combinatorial way, as the exponential growth of the number of paths with fixed endpoints [8].

Proposition 1.2 (Gurevich). Let $G$ be a connected oriented graph. Then for any vertices $u, v$

$$
h(G)=\lim _{n \rightarrow+\infty} \frac{1}{n} \log p_{u v}^{G}(n)=-\log R(G) .
$$

Another way to compute the entropy is to compactify the space $\Gamma_{G}$ and then use the definition of topological entropy for compact metric spaces. If $G$ is an oriented graph, denote the one-point compactification of $V(G)$ by $V(G) \cup\{\infty\}$ and define $\bar{\Gamma}_{G}$ as the closure of $\Gamma_{G}$ in $(V(G) \cup\{\infty\})^{\mathbb{Z}}$. The distance $d$ naturally extends to $\bar{\Gamma}_{G}$. In [7] Gurevich shows that this gives the same entropy; this means that there is only very little dynamics added in this compactification. Moreover, the Variational Principle is still valid for Markov chains [7].

Theorem 1.3 (Gurevich). Let $G$ be an oriented graph. Then

$$
h(G)=h_{\mathrm{top}}\left(\bar{\Gamma}_{G}, \sigma\right)=\sup \left\{h_{\mu}\left(\Gamma_{G}\right) \mid \mu \sigma \text {-invariant probability measure }\right\} .
$$

\section{On the classification of connected graphs.}

2.1. Transient, null recurrent, positive recurrent graphs. In $[16]$ Vere-Jones gives a classification of connected graphs as transient, null recurrent or positive recurrent. The definitions are given in Table 1 (lines 1 and 2) as well as properties of the series $\sum p_{u v}^{G}(n) z^{n}$ which give an alternative definition.

In [13] and [14] Salama studies the links between the classification and the possibility to extend or contract a graph without changing its entropy. It follows that a connected graph is transient if and only if it is strictly included in a connected graph of equal entropy, and that a graph with no proper subgraph of equal entropy is positive recurrent.

Remark 2.1. In [13] Salama claims that $L_{u u}$ is independent of $u$, which is not true; in [14] he uses the quantity $L=\inf _{u} L_{u u}$ and he states that if $R=L$ then $R=L_{u u}$ for all vertices $u$, which is wrong too (see Proposition 3.2 in $[\mathbf{9}])$. It follows that in $[\mathbf{1 3}]$ and $[\mathbf{1 4}]$ the statement " $\mathrm{R}=\mathrm{L}$ " must be interpreted either as " $R=L_{u u}$ for some $u$ " or " $R=L_{u u}$ for all $u$ " depending on the context. This encouraged us to give the proofs of Salama's results in this article. 


\begin{tabular}{l|c|c|c} 
& transient & $\begin{array}{c}\text { null } \\
\text { recurrent }\end{array}$ & $\begin{array}{c}\text { positive } \\
\text { recurrent }\end{array}$ \\
\hline$\sum_{n>0} f_{u u}^{G}(n) R^{n}$ & $<1$ & 1 & 1 \\
\hline$\sum_{n>0} n f_{u u}^{G}(n) R^{n}$ & $\leq+\infty$ & $+\infty$ & $<+\infty$ \\
\hline$\sum_{n \geq 0} p_{u v}^{G}(n) R^{n}$ & $<+\infty$ & $+\infty$ & $+\infty$ \\
\hline $\lim _{n \rightarrow+\infty} p_{u v}^{G}(n) R^{n}$ & 0 & 0 & $\lambda_{u v}>0$ \\
\hline & $R=L_{u u}$ & $R=L_{u u}$ & $R \leq L_{u u}$
\end{tabular}

Table 1. Properties of the series associated to a transient, null recurrent or positive recurrent graph $G$; these properties do not depend on the vertices $u, v$ ( $G$ is connected).

In [14] Salama shows that a transient or null recurrent graph satisfies $R=L_{u u}$ for all vertices $u$; we give the unpublished proof due to U. Fiebig $[6]$.

Proposition 2.2 (Salama). Let $G$ be a connected oriented graph. If $G$ is transient or null recurrent then $R=L_{u u}$ for all vertices $u$. Equivalently, if there exists a vertex $u$ such that $R<L_{u u}$ then $G$ is positive recurrent.

Proof. For a connected oriented graph, it is obvious that $R \leq L_{u u}$ for all $u$, thus the two claims of the Proposition are equivalent. We prove the second one.

Let $u$ be a vertex of $G$ such that $R<L_{u u}$. Let $F(x)=\sum_{n \geq 1} f_{u u}^{G}(n) x^{n}$ for all $x \geq 0$. If we break a loop based in $u$ into first return loops, we get the following formula:

$$
\sum_{n \geq 0} p_{u u}^{G}(n) x^{n}=\sum_{k \geq 0}(F(x))^{k}
$$

Suppose that $G$ is transient, that is, $F(R)<1$. The map $F$ is analytic on $\left[0, L_{u u}\right)$ and $R<L_{u u}$ thus there exists $R<x<L_{u u}$ such that $F(x)<$ 1. According to Equation (1) one gets that $\sum_{n \geq 0} p_{u u}^{G}(n) x^{n}<+\infty$, which contradicts the definition of $R$. Therefore $G$ is recurrent. Moreover $R<$ $L_{u u}$ by assumption, thus $\sum_{n \geq 1} n f_{u u}^{G}(n) R^{n}<+\infty$, which implies that $G$ is positive recurrent.

Definition 2.3. A connected oriented graph is called strongly positive recurrent if $R<L_{u u}$ for all vertices $u$.

Lemma 2.4. Let $G$ be a connected oriented graph and $u$ a vertex.

i) $R<L_{u u}$ if and only if $\sum_{n \geq 1} f_{u u}^{G}(n) L_{u u}^{n}>1$. 
ii) If $G$ is recurrent then $R$ is the unique positive number $x$ such that $\sum_{n \geq 1} f_{u u}^{G}(n) x^{n}=1$.

Proof. Use the fact that $F(x)=\sum_{n \geq 1} f_{u u}^{G}(n) x^{n}$ is increasing.

The following result deals with transient graphs [13]:

Theorem 2.5 (Salama). Let $G$ be a connected oriented graph of finite positive entropy. Then $G$ is transient if and only if there exists a connected oriented graph $G^{\prime} \nsupseteq G$ such that $h\left(G^{\prime}\right)=h(G)$. If $G$ is transient then $G^{\prime}$ can be chosen transient.

Proof. The assumption on the entropy implies that $0<R<1$. Suppose first that there exists a connected graph $G^{\prime} \nsupseteq G$ such that $h\left(G^{\prime}\right)=h(G)$, that is, $R^{\prime}=R$. Fix a vertex $u$ in $G$. The graph $G$ is a proper subgraph of $G^{\prime}$ thus there exists $n$ such that $f_{u u}^{G}(n)<f_{u u}^{G^{\prime}}(n)$, which implies that

$$
\sum_{n \geq 1} f_{u u}^{G}(n) R^{n}<\sum f_{u u}^{G^{\prime}}(n) R^{\prime n} \leq 1 .
$$

Therefore $G$ is transient.

Now suppose that $G$ is transient and fix a vertex $u$ in $G$. One has $\sum_{n \geq 1} f_{u u}^{G}(n) R^{n}<1$. Let $k \geq 2$ be an integer such that

$$
\sum_{n \geq 1} f_{u u}^{G}(n) R^{n}+R^{k}<1 .
$$

Define the graph $G^{\prime}$ by adding a loop of length $k$ based at the vertex $u$; one has $R^{\prime} \leq R$ and

$$
\sum_{n \geq 1} f_{u u}^{G^{\prime}}(n) R^{\prime n} \leq \sum_{n \geq 1} f_{u u}^{G^{\prime}}(n) R^{n}=\sum_{n \geq 1} f_{u u}^{G}(n) R^{n}+R^{k}<1 .
$$

Equation (2) implies that $R \leq L_{u u}^{\prime}$ and also that the graph $G^{\prime}$ is transient, so $R^{\prime}=L_{u u}^{\prime}$ by Proposition 2.2. Then one has $L_{u u}^{\prime}=R^{\prime} \leq R \leq L_{u u}^{\prime}$ thus $R=R^{\prime}$.

In [14] Salama proves that if $R=L_{u u}$ for all vertices $u$ then there exists a proper subgraph of equal entropy. We show that the same conclusion holds if one supposes that $R=L_{u u}$ for some $u$. The proof below is a variant of the one of Salama. The converse is also true, as shown by U. Fiebig [6].

Proposition 2.6. Let $G$ be a connected oriented graph of positive entropy.

i) If there is a vertex $u$ such that $R=L_{u u}$ then there exists a connected subgraph $G^{\prime} \varsubsetneqq G$ such that $h\left(G^{\prime}\right)=h(G)$.

ii) If there is a vertex $u$ such that $R<L_{u u}$ then for all proper subgraphs $G^{\prime}$ one has $h\left(G^{\prime}\right)<h(G)$. 
Proof. i) Suppose that $R=L_{u u}$. If $u_{0}=u$ is followed by a unique vertex, let $u_{1}$ be this vertex. If $u_{1}$ is followed by a unique vertex, let $u_{2}$ be this vertex, and so on. If this leads to define $u_{n}$ for all $n$ then $h(G)=0$, which is not allowed.

Let $u_{k}$ be the last built vertex; there exist two distinct vertices $v, v^{\prime}$ such that $u_{k} \rightarrow v$ and $u_{k} \rightarrow v^{\prime}$. Let $G_{1}^{\prime}$ be the graph $G$ deprived of the arrow $u_{k} \rightarrow v$ and $G_{2}^{\prime}$ the graph $G$ deprived of all the arrows $u_{k} \rightarrow w, w \neq v$. Call $G_{i}$ the connected component of $G_{i}^{\prime}$ that contains $u(i=1,2)$; obviously $G_{i} \varsubsetneqq G$. For all $n \geq 1$ one has

$$
f_{u u}^{G}(n)=f_{u_{k} u}^{G}(n-k)=f_{u_{k} u}^{G_{1}}(n-k)+f_{u_{k} u}^{G_{2}}(n-k),
$$

thus there exists $i \in\{1,2\}$ such that $L_{u u}=L_{u_{k} u}\left(G_{i}\right)$. One has

$$
R \leq R\left(G_{i}\right) \leq L_{u_{k} u}\left(G_{i}\right)=L_{u u}=R,
$$

thus $R=R\left(G_{i}\right)$, that is, $h(G)=h\left(G_{i}\right)$.

ii) Suppose that $R<L_{u u}$ and consider $G^{\prime} \varsubsetneqq G$. Suppose first that $u$ is a vertex of $G^{\prime}$. The graph $G$ is positive recurrent by Proposition 2.2 so $\sum_{n \geq 1} f_{u u}^{G}(n) R^{n}=1$. Since $G^{\prime} \varsubsetneqq G$ there exists $n$ such that $f_{u u}^{G^{\prime}}(n)<f_{u u}^{G}(n)$, thus

$$
\sum_{n \geq 1} f_{u u}^{G^{\prime}} R^{n}<1
$$

Moreover $L_{u u}^{\prime} \geq L_{u u}$. If $G^{\prime}$ is transient then $R^{\prime}=L_{u u}^{\prime}$ (Proposition 2.2) thus $R^{\prime} \geq L_{u u}>R$. If $G^{\prime}$ is recurrent then $\sum_{n>1} f_{u u}^{G^{\prime}} R^{\prime n}=1$ thus $R^{\prime}>R$ because of Equation (3). In both cases $R^{\prime}>R$, that is, $h\left(G^{\prime}\right)<h(G)$.

Suppose now that $u$ is not a vertex of $G^{\prime}$ and fix a vertex $v$ in $G^{\prime}$. Let $\left(u_{0}, \ldots, u_{p}\right)$ a path (in $G$ ) of minimal length between $u=u_{0}$ and $v=u_{p}$, and let $\left(v_{0}, \ldots, v_{q}\right)$ be a path of minimal length between $v=v_{0}$ and $u=v_{q}$.

If $\left(w_{0}=v, w_{1}, \ldots, w_{n}=v\right)$ is a loop in $G^{\prime}$ then

$$
\left(u_{0}=u, u_{1}, \ldots, u_{p}=w_{0}, w_{1}, \ldots, w_{n}=v_{0}, v_{1}, \ldots, v_{q}=u\right)
$$

is a first return loop based in $u$ in the graph $G$. For all $n \geq 0$ we get that $p_{v v}^{G^{\prime}}(n) \leq f_{u u}^{G}(n+p+q)$, thus $R^{\prime} \geq L_{u u}>R$, that is, $h\left(G^{\prime}\right)<h(G)$.

The following result gives a characterization of strongly positive recurrent graphs. It is a straightforward corollary of Proposition 2.6 (see also [6]).

Theorem 2.7. Let $G$ be a connected oriented graph of positive entropy. The following properties are equivalent:

i) For all $u$ one has $R<L_{u u}$ (that is, $G$ is strongly positive recurrent),

ii) there exists $u$ such that $R<L_{u u}$,

iii) $G$ has no proper subgraph of equal entropy. 
2.2. Recurrent extensions of equal entropy of transient graphs. We show that any transient graph $G$ can be extended to a recurrent graph without changing the entropy by adding a (possibly infinite) number of loops. If the series $\sum n f_{v v}^{G}(n) R^{n}$ is finite then the obtained recurrent graph is positive recurrent (but not strongly positive recurrent), otherwise it is null recurrent.

Proposition 2.8. Let $G$ be a transient graph of finite positive entropy. Then there exists a recurrent graph $G^{\prime} \supset G$ such that $h(G)=h\left(G^{\prime}\right)$. Moreover $G^{\prime}$ can be chosen to be positive recurrent if $\sum_{n>0} n f_{u u}^{G}(n) R^{n}<+\infty$ for some vertex $u$ of $G$, and $G^{\prime}$ is necessarily null recurrent otherwise.

Proof. The entropy of $G$ is finite and positive thus $0<R<1$ and there exists an integer $p$ such that $\frac{1}{2} \leq p R<1$. Define $\alpha=p R$. Let $u$ be a vertex of $G$ and define $D=1-\sum_{n \geq 1} f_{u u}^{G}(n) R^{n}$; one has $0<D<1$. Moreover

$$
\sum_{n \geq 1} \alpha^{n} \geq \sum_{n \geq 1} \frac{1}{2^{n}}=1
$$

thus

$$
\sum_{n \geq k+1} \alpha^{n}=\alpha^{k} \sum_{n \geq 1} \alpha^{n} \geq \alpha^{k}
$$

We build a sequence of integers $\left(n_{i}\right)_{i \in I}$ such that $2 \sum_{i \in I} \alpha^{n_{i}}=D$. For this, we define inductively a strictly increasing (finite or infinite) sequence of integers $\left(n_{i}\right)_{i \in I}$ such that for all $k \in I$

$$
\sum_{i=0}^{k} \alpha^{n_{i}} \leq \frac{D}{2}<\sum_{i=0}^{k} \alpha^{n_{i}}+\sum_{n>n_{k}} \alpha^{n} .
$$

- Let $n_{0}$ be the greatest integer $n \geq 2$ such that $\sum_{k \geq n} \alpha^{k}>\frac{D}{2}$. By choice of $n_{0}$ one has $\sum_{n \geq n_{0}+1} \alpha^{n} \leq \frac{D}{2}$, thus $\alpha^{n_{0}} \leq \frac{D}{2}$ by Equation (4). This is the required property at rank 0 .

- Suppose that $\left(n_{0}, \ldots, n_{k}\right)$ is already defined. If $\sum_{i=0}^{k} \alpha^{n_{i}}=\frac{D}{2}$ then $I=\{0, \ldots, k\}$ and we stop the construction. Otherwise let $n_{k+1}$ be the greatest integer $n>n_{k}$ such that

$$
\sum_{i=0}^{k} \alpha^{n_{i}}+\sum_{j \geq n} \alpha^{j}>\frac{D}{2} .
$$

By choice of $n_{k+1}$ and Equation (4), one has

$$
\alpha^{n_{k+1}} \leq \sum_{j \geq n_{k+1}+1} \alpha^{j} \leq \frac{D}{2}-\sum_{i=0}^{k} \alpha^{n_{i}} .
$$

This is the required property at rank $k+1$. 
Define a new graph $G^{\prime} \supset G$ by adding $2 p^{n_{i}}$ loops of length $n_{i}$ based at the vertex $u$. Obviously one has $R^{\prime} \leq R$, and $\sum_{i \in I}(p R)^{n_{i}}=\frac{D}{2}$ by construction. Therefore

$$
\sum_{n \geq 1} f_{u u}^{G^{\prime}}(n) R^{n}=\sum_{n \geq 1} f_{u u}^{G}(n) R^{n}+\sum_{i \in I} 2(p R)^{n_{i}}=1 .
$$

This implies that $R \leq L_{u u}^{\prime}$. If $G^{\prime}$ is transient then $\sum_{n>1} f_{u u}^{G^{\prime}}(n) R^{\prime n}<1$ and $R^{\prime}=L_{u u}^{\prime}$ by Proposition 2.2, thus $R \leq R^{\prime}$ and Equation (5) leads to a contradiction. Therefore $G^{\prime}$ is recurrent. By Lemma 2.4(ii) one has $R^{\prime}=R$, that is, $h\left(G^{\prime}\right)=h(G)$. In addition,

$$
\sum_{n \geq 1} n f_{u u}^{G^{\prime}}(n) R^{n}=\sum_{n \geq 1} n f_{u u}^{G}(n) R^{n}+\sum_{i \in I} n_{i} \alpha^{n_{i}}
$$

and this quantity is finite if and only if $\sum n f_{u u}^{G}(n) R^{n}$ is finite. In this case the graph $G^{\prime}$ is positive recurrent.

If $\sum n f_{u u}^{G}(n) R^{n}=+\infty$, let $H$ be a recurrent graph containing $G$ with $h(H)=h(G)$. Then $H$ is null recurrent because

$$
\sum_{n \geq 1} n f_{u u}^{H}(n) R^{n} \geq \sum_{n \geq 1} n f_{u u}^{G}(n) R^{n}=+\infty .
$$

Example 2.9. We build a positive (resp. null) recurrent graph $G$ such that $\sum f_{u u}^{G}(n) L_{u u}^{n}=1$ and then we delete an arrow to obtain a graph $G^{\prime} \subset G$ which is transient and such that $h\left(G^{\prime}\right)=h(G)$. First we give a description of $G$ depending on a sequence of integers $a(n)$ then we give two different values to the sequence $a(n)$ so as to obtain a positive recurrent graph in one case and a null recurrent graph in the other case.

Let $u$ be a vertex and $a(n)$ a sequence of nonnegative integers for $n \geq 1$, with $a(1)=1$. The graph $G$ is composed of $a(n)$ loops of length $n$ based at the vertex $u$ for all $n \geq 1$ (see Figure 1). More precisely, define the set of vertices of $G$ as

$$
V=\{u\} \cup \bigcup_{n=1}^{+\infty}\left\{v_{k}^{n, i} \mid 1 \leq i \leq a(n), 1 \leq k \leq n-1\right\},
$$

where the vertices $v_{k}^{n, i}$ above are distinct. Let $v_{0}^{n, i}=v_{n}^{n, i}=u$ for $1 \leq i \leq$ $a(n)$. There is an arrow $v_{k}^{n, i} \rightarrow v_{k+1}^{n, i}$ for $0 \leq k \leq n-1,1 \leq i \leq a(n), n \geq 1$ and there is no other arrow in $G$. The graph $G$ is connected and $f_{u u}^{G}(n)=$ $a(n)$ for $n \geq 1$.

The sequence $(a(n))_{n \geq 2}$ is chosen such that it satisfies

$$
\sum_{n \geq 1} a(n) L^{n}=1
$$




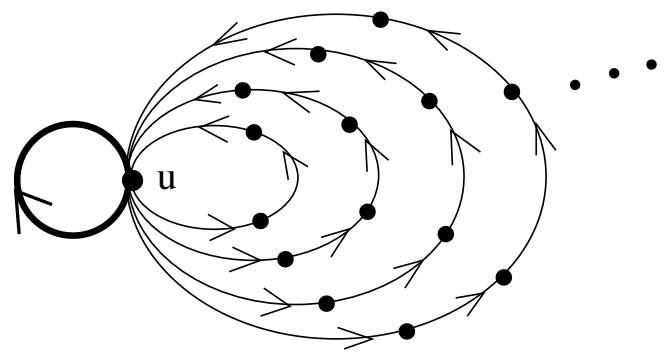

Figure 1. The graphs $G$ and $G^{\prime}$; the bold loop (on the left) is the only arrow that belongs to $G$ and not to $G^{\prime}$, otherwise the two graphs coincide.

where $L=L_{u u}>0$ is the radius of convergence of the series $\sum a(n) z^{n}$. If $G$ is transient then $R=L_{u u}$ by Proposition 2.2, but Equation (6) contradicts the definition of transient. Thus $G$ is recurrent. Moreover, $R=L$ by Lemma 2.4(ii).

The graph $G^{\prime}$ is obtained from $G$ by deleting the arrow $u \rightarrow u$. Obviously one has $L_{u u}^{\prime}=L$ and

$$
\sum_{n \geq 1} f_{u u}^{G^{\prime}}(n) L^{n}=1-L<1 .
$$

This implies that $G^{\prime}$ is transient because $R^{\prime} \leq L_{u u}^{\prime}$. Moreover $R^{\prime}=L_{u u}^{\prime}$ by Proposition 2.2 thus $R^{\prime}=R$, that is, $h\left(G^{\prime}\right)=h(G)$.

Now we consider two different sequences $a(n)$.

1) Let $a\left(n^{2}\right)=2^{n^{2}-n}$ for $n \geq 1$ and $a(n)=0$ otherwise. Then $L=\frac{1}{2}$ and

$$
\sum_{n \geq 1} f_{u u}^{G}(n) L^{n}=\sum_{n \geq 1} 2^{n^{2}-n} \frac{1}{2^{n^{2}}}=\sum_{n \geq 1} \frac{1}{2^{n}}=1 .
$$

Moreover

$$
\sum_{n \geq 1} n f_{u u}^{G}(n) L^{n}=\sum_{n \geq 1} \frac{n^{2}}{2^{n}}<+\infty,
$$

hence the graph $G$ is positive recurrent.

2) Let $a(1)=1, a\left(2^{n}\right)=2^{2^{n}-n}$ for $n \geq 2$ and $a(n)=0$ otherwise. One can compute that $L=\frac{1}{2}$, and

$$
\sum_{n \geq 1} f_{u u}^{G}(n) L^{n}=\frac{1}{2}+\sum_{n \geq 2} 2^{2^{n}-n} \frac{1}{2^{2^{n}}}=\frac{1}{2}+\sum_{n \geq 2} \frac{1}{2^{n}}=1 .
$$

Moreover

$$
\sum_{n \geq 1} n f_{u u}^{G}(n) L^{n}=\frac{1}{2}+\sum_{n \geq 2} 2^{n} \frac{1}{2^{n}}=+\infty
$$


hence the graph $G$ is null recurrent.

Remark 2.10. Let $G$ be a transient graph of finite entropy. Fix a vertex $u$ and choose an integer $k$ such that $\sum_{n \geq k} R^{n}<1-\sum_{n \geq 1} f_{u u}^{G}(n) R^{n}$. For every integer $n \geq k$ let $m_{n}=\left\lfloor R^{-n}\right\rfloor$, add $\left\lfloor R^{-\left(m_{n}-n\right)}\right\rfloor$ loops of length $m_{n}$ based at the vertex $u$ and call $G^{\prime}$ the graph obtained in this way. It can be shown that the graph $G^{\prime}$ is transient, $h\left(G^{\prime}\right)=h(G)$ and $\sum_{n \geq 1} n f_{u u}^{G^{\prime}} R^{\prime n}=+\infty$. Then Proposition 2.8 implies that every transient graph is included in a null recurrent graph of equal entropy.

Remark 2.11. In the more general setting of thermodynamic formalism for countable Markov chains, Sarig puts to the fore a subclass of positive recurrent potentials which he calls strongly positive recurrent [15]; his motivation is different, but the classifications agree. If $G$ is a countable oriented graph, a potential is a continuous map $\phi: \Gamma_{G} \rightarrow \mathbb{R}$ and the pressure $P(\phi)$ is the analogous of the Gurevich entropy, the paths being weighted by $e^{\phi}$; a potential is either transient or null recurrent or positive recurrent. Considering the null potential $\phi \equiv 0$, we retrieve the case of (non-weighted) topological Markov chains. In [15] Sarig introduces a quantity $\Delta_{u}[\phi] ; \phi$ is transient (resp. recurrent) if $\Delta_{u}[\phi]<0$ (resp. $\Delta_{u}[\phi] \geq 0$ ). The potential is called strongly positive recurrent if $\Delta_{u}[\phi]>0$, which implies it is positive recurrent. A strongly positive recurrent potential $\phi$ is stable under perturbation, that is, any potential $\phi+t \psi$ close to $\phi$ is positive recurrent too. For the null potential, $\Delta_{u}[0]=\log \left(\sum_{n \geq 1} f_{u u}^{G}(n) L^{n}\right)$, thus $\Delta_{u}[0]>0$ if and only if the graph is strongly positive recurrent (Lemma 2.4 and Theorem 2.7). In [9] strongly positive recurrent potentials are called stable positive.

Examples of (non-null) potentials which are positive recurrent but not strongly positive recurrent can be found in [15]; some of them resemble much the Markov chains of Example 2.9, their graphs being composed of loops as in Figure 1.

\section{Existence of a maximal measure.}

3.1. Positive recurrence and maximal measures. A Markov chain on a finite graph always has a maximal measure [12], but it is not the case for infinite graphs [7]. In [8] Gurevich gives a necessary and sufficient condition for the existence of such a measure.

Theorem 3.1 (Gurevich). Let $G$ be a connected oriented graph of finite positive entropy. Then the Markov chain $\left(\Gamma_{G}, \sigma\right)$ admits a maximal measure if and only if the graph is positive recurrent. Moreover, such a measure is unique if it exists, and it is an ergodic Markov measure.

In [10] Gurevich and Zargaryan show that if one can find a finite connected subgraph $H \subset G$ such that there are more paths inside than outside 
$H$ (in term of exponential growth), then the graph $G$ has a maximal measure. This condition is equivalent to strong positive recurrent as it was shown by Gurevich and Savchenko in the more general setting of weighted graphs $[\mathbf{9}]$.

Let $G$ be a connected oriented graph, $W$ a subset of vertices and $u, v$ two vertices of $G$. Define $t_{u v}^{W}(n)$ as the number of paths $\left(v_{0}, \ldots, v_{n}\right)$ such that $v_{0}=u, v_{n}=v$ and $v_{i} \in W$ for all $0<i<n$, and put $\tau_{u v}^{W}=$ $\limsup _{n \rightarrow+\infty} \frac{1}{n} \log t_{u v}^{W}(n)$.

Theorem 3.2 (Gurevich-Zargaryan). Let $G$ be a connected oriented graph of finite positive entropy. If there exists a finite set of vertices $W$ such that $W$ is connected and for all vertices $u, v$ in $W, \tau_{u v}^{\bar{W}} \leq h(W)$, then the graph $G$ is strongly positive recurrent.

For graphs that are not strongly positive recurrent the entropy is mainly concentrated near infinity in the sense that it is supported by the infinite paths that spend most of the time outside a finite subgraph (Proposition 3.3). This result is obtained by applying inductively the construction of Proposition 2.6(i). As a corollary, there exist "almost maximal measures escaping to infinity" (Corollary 3.4). These two results are proven and used as tools to study interval maps in [4], but they are interesting by themselves, that is why we state them here.

Proposition 3.3. Let $G$ be a connected oriented graph which is not strongly positive recurrent and $W$ a finite set of vertices. Then for all integers $n$ there exists a connected subgraph $G_{n} \subset G$ such that $h\left(G_{n}\right)=h(G)$ and for all $w \in W$, for all $0 \leq k<n, f_{w w}^{G_{n}}(k)=0$.

Corollary 3.4. Let $G$ be a connected oriented graph which is not strongly positive recurrent. Then there exists a sequence of ergodic Markov measures $\left(\mu_{n}\right)_{n \geq 0}$ such that $\lim _{n \rightarrow+\infty} h_{\mu_{n}}\left(\Gamma_{G}, \sigma\right)=h(G)$ and for all finite subsets of vertices $W, \lim _{n \rightarrow+\infty} \mu_{n}\left(\left\{\left(u_{n}\right)_{n \in \mathbb{Z}} \in \Gamma_{G} \mid u_{0} \in W\right\}\right)=0$.

3.2. Local entropy and maximal measures. For a compact system, the local entropy is defined according to a distance but does not depend on it. One may wish to extend this definition to noncompact metric spaces although the notion obtained in this way is not canonical.

Definition 3.5. Let $X$ be a metric space, $d$ its distance and let $T: X \rightarrow X$ be a continuous map.

The Bowen ball of centre $x$, of radius $r$ and of order $n$ is defined as

$$
B_{n}(x, r)=\left\{y \in X \mid d\left(T^{i} x, T^{i} y\right)<r, 0 \leq i<n\right\} .
$$

$E$ is a $(\delta, n)$-separated set if

$$
\forall y, y^{\prime} \in E, y \neq y^{\prime}, \exists 0 \leq k<n, d\left(T^{k} y, T^{k} y^{\prime}\right) \geq \delta .
$$


The maximal cardinality of a $(\delta, n)$-separated set contained in $Y$ is denoted by $s_{n}(\delta, Y)$.

The local entropy of $(X, T)$ is defined as $h_{\mathrm{loc}}(X)=\lim _{\varepsilon \rightarrow 0} h_{\mathrm{loc}}(X, \varepsilon)$, where

$$
h_{\mathrm{loc}}(X, \varepsilon)=\lim _{\delta \rightarrow 0} \limsup _{n \rightarrow+\infty} \frac{1}{n} \sup _{x \in X} \log s_{n}\left(\delta, B_{n}(x, \varepsilon)\right) .
$$

If the space $X$ is not compact, these notions depend on the distance. When $X=\Gamma_{G}$, we use the distance $d$ introduced in Section 1.2. The local entropy of $\Gamma_{G}$ does not depend on the identification of the vertices with $\mathbb{N}$.

Proposition 3.6. Let $\Gamma_{G}$ be the topological Markov chain on $G$ and $\bar{\Gamma}_{G}$ its compactification as defined in Section 1.2. Then $h_{\mathrm{loc}}\left(\Gamma_{G}\right)=h_{\mathrm{loc}}\left(\bar{\Gamma}_{G}\right)$.

Proof. Let $\bar{u}=\left(u_{n}\right)_{n \in \mathbb{Z}} \in \bar{\Gamma}_{G}, \varepsilon>0$ and $k \geq 1$. By continuity there exists $\eta>0$ such that, if $\bar{v} \in \bar{\Gamma}_{G}$ and $d(\bar{u}, \bar{v})<\eta$ then $d\left(\sigma^{i}(\bar{u}), \sigma^{i}(\bar{v})\right)<\varepsilon$ for all $0 \leq i<k$. By definition of $\bar{\Gamma}_{G}$ there is $\bar{v} \in \Gamma_{G}$ such that $d(\bar{u}, \bar{v})<\eta$, thus $\bar{u} \in B_{k}(\bar{v}, \varepsilon)$, which implies that $B_{k}(\bar{u}, \varepsilon) \subset B_{k}(\bar{v}, 2 \varepsilon)$. Consequently $h_{\text {loc }}\left(\bar{\Gamma}_{G}, \varepsilon\right) \leq h_{\text {loc }}(\Gamma, 2 \varepsilon)$, and $h_{\text {loc }}\left(\bar{\Gamma}_{G}\right) \leq h_{\text {loc }}\left(\Gamma_{G}\right)$. The reverse inequality is obvious.

We are going to prove that, if $h_{\text {loc }}\left(\Gamma_{G}\right)<h(G)$, then $G$ is strongly positive recurrent. First we introduce some notations.

Let $G$ be an oriented graph. If $V$ is a subset of vertices, $H$ a subgraph of $G$ and $\bar{u}=\left(u_{n}\right)_{n \in \mathbb{Z}} \in \Gamma_{G}$, define

$$
\begin{aligned}
& \mathcal{C}^{H}(\bar{u}, V) \\
& \quad=\left\{\left(v_{n}\right)_{n \in \mathbb{Z}} \in \Gamma_{H} \mid \forall n \in \mathbb{Z}, u_{n} \in V \Rightarrow\left(v_{n}=u_{n}\right), u_{n} \notin V \Rightarrow v_{n} \notin V\right\} .
\end{aligned}
$$

If $S \subset \Gamma_{G}$ and $p, q \in \mathbb{Z} \cup\{-\infty,+\infty\}$, define

$$
[S]_{p}^{q}=\left\{\left(v_{n}\right)_{n \in \mathbb{Z}} \in \Gamma_{G} \mid \exists\left(u_{n}\right)_{n \in \mathbb{Z}} \in S, \forall p \leq n \leq q, u_{n}=v_{n}\right\} .
$$

Lemma 3.7. Let $G$ be an oriented graph on the set of vertices $\mathbb{N}$.

i) If $V \supset\{0, \ldots, p+2\}$ then for all $\bar{u} \in \Gamma_{G}$ and all $n \geq 1, \mathcal{C}^{G}(\bar{u}, V) \subset$ $B_{n}\left(\bar{u}, 2^{-p}\right)$.

ii) If $\bar{u}=\left(u_{n}\right)_{n \in \mathbb{Z}}$ and $\bar{v}=\left(v_{n}\right)_{n \in \mathbb{Z}}$ are two paths in $G$ such that $\left(u_{0}, \ldots\right.$, $\left.u_{n-1}\right) \neq\left(v_{0}, \ldots, v_{n-1}\right)$ and $u_{i}, v_{i} \in\{0, \ldots, q-1\}$ for $0 \leq i \leq n-1$ then $(\bar{u}, \bar{v})$ is $\left(2^{-q}, n\right)$-separated.

Proof. (i) Let $\bar{u}=\left(u_{n}\right)_{n \in \mathbb{Z}} \in \Gamma_{G}$. If $\bar{v}=\left(v_{n}\right)_{n \in \mathbb{Z}} \in \mathcal{C}^{G}(\bar{u}, V)$, then $D\left(u_{j}, v_{j}\right) \leq 2^{-(p+2)}$ for all $j \in \mathbb{Z}$. Consequently for all $0 \leq i<n$

$$
d\left(\sigma^{i}(\bar{u}), \sigma^{i}(\bar{v})\right)=\sum_{k \in \mathbb{Z}} \frac{D\left(u_{i+k}, v_{i+k}\right)}{2^{|k|}} \leq \sum_{k \in \mathbb{Z}} \frac{2^{-(p+2)}}{2^{|k|}} \leq 3 \cdot 2^{-(p+2)}<2^{-p} .
$$


(ii) Let $0 \leq i \leq n-1$ such that $u_{i} \neq v_{i}$. By hypothesis, $u_{i}, v_{i} \leq q-1$. Suppose that $u_{i}<v_{i}$. Then $d\left(\sigma^{i}(\bar{u}), \sigma^{i}(\bar{v})\right) \geq D\left(u_{i}, v_{i}\right)=2^{-u_{i}}\left(1-2^{-\left(v_{i}-u_{i}\right)}\right) \geq$ $2^{-q}$.

Theorem 3.8. Let $G$ be a connected oriented graph of finite entropy on the set of vertices $\mathbb{N}$. If $h_{\mathrm{loc}}\left(\Gamma_{G}\right)<h(G)$, then the graph $G$ is strongly positive recurrent and the Markov chain $\left(\Gamma_{G}, \sigma\right)$ admits a maximal measure.

Proof. Fix $C$ and $\varepsilon>0$ such that $h_{\mathrm{loc}}\left(\Gamma_{G}, \varepsilon\right)<C<h(G)$. Let $p$ be an integer such that $2^{-(p-1)}<\varepsilon$. Let $G^{\prime}$ be a finite subgraph such that $h\left(G^{\prime}\right)>C$ and let $V$ be a finite subset of vertices such that $V$ is connected and contains the vertices of $G^{\prime}$ and the vertices $\{0, \ldots, p\}$. Define $W=\bar{V}$, $V_{q}=\{n \leq q\}$ and $W_{q}=V_{q} \backslash V=W \cap V_{q}$ for all $q \geq 1$.

Our aim is to bound $t_{u u^{\prime}}^{W}(n)=t_{u u^{\prime}}^{\bar{V}}(n)$. Choose $u, u^{\prime} \in V$ and let $\left(w_{0}, \ldots\right.$, $w_{n_{0}}$ ) be a path between $u^{\prime}$ and $u$ with $w_{i} \in V$ for $0 \leq i \leq n_{0}$. Fix $n \geq 1$. One has $t_{u u^{\prime}}^{W}(n)=\lim _{q \rightarrow+\infty} t_{u u^{\prime}}^{W_{q}}(n)$.

Fix $\delta_{0}>0$ such that

$$
\forall \delta \leq \delta_{0}, \limsup _{n \rightarrow+\infty} \frac{1}{n} \sup _{\bar{v} \in \Gamma_{G}} \log s_{n}\left(\delta, B_{n}(\bar{v}, \varepsilon)\right)<C .
$$

Take $q \geq 1$ arbitrarily large and $\delta \leq \min \left\{\delta_{0}, 2^{-(q+1)}\right\}$. Choose $N$ such that

$$
\forall n \geq N, \forall \bar{v} \in \Gamma_{G}, \frac{1}{n} \log s_{n}\left(\delta, B_{n}(\bar{v}, \varepsilon)\right)<C .
$$

If $t_{u u^{\prime}}^{W_{q}}(n) \neq 0$, choose a path $\left(v_{0}, \ldots, v_{n}\right)$ such that $v_{0}=u, v_{n}=u^{\prime}$ and $v_{i} \in W_{q}$ for $0<i<q$. Define $\bar{v}^{(n)}=\left(v_{i}^{(n)}\right)_{i \in \mathbb{Z}}$ as the periodic path of period $n+n_{0}$ satisfying $v_{i}^{(n)}=v_{i}$ for $0 \leq i \leq n$ and $v_{n+i}^{(n)}=w_{i}$ for $0 \leq i \leq n_{0}$.

Define the set $E_{q}(n, k)$ as follows (see Figure 2):

$$
E_{q}(n, k)=\left[\mathcal{C}^{V_{q}}\left(\bar{v}^{(n)}, V\right)\right]_{0}^{k\left(n+n_{0}\right)} \cap\left[\bar{v}^{(n)}\right]_{-\infty}^{0} \cap\left[\bar{v}^{(n)}\right]_{k\left(n+n_{0}\right)}^{+\infty} .
$$

The paths in $E_{q}(n, 1)$ are exactly the paths counted by $t_{u u^{\prime}}^{W_{q}}(n)$ which are extended outside the indices $\{0, \ldots, n\}$ like the path $\bar{v}^{(n)}$, thus $\# E_{q}(n, 1)=$ $t_{u u^{\prime}}^{W_{q}}(n)$. Similarly, \# $E_{q}(n, k)=\left(t_{u u^{\prime}}^{W_{q}}(n)\right)^{k}$.

By definition, $E_{q}(n, k) \subset \mathcal{C}^{G}\left(\bar{v}^{(n)}, V\right)$ and $\{0, \ldots, p\} \subset V$ thus $E_{q}(n, k) \subset$ $B_{k\left(n+n_{0}\right)}\left(\bar{v}^{(n)}, \varepsilon\right)$ by Lemma $3.7(\mathrm{i})$. Moreover, if $\left(w_{i}\right)_{i \in \mathbb{Z}}$ and $\left(w_{i}^{\prime}\right)_{i \in \mathbb{Z}}$ are two distinct elements of $E_{q}(n, k)$, there exists $0 \leq i<k\left(n+n_{0}\right)$ such that $w_{i} \neq w_{i}^{\prime}$ and $w_{i}, w_{i}^{\prime} \leq q$, thus $E_{q}(n, k)$ is a $\left(\delta, k\left(n+n_{0}\right)\right)$-separated set by Lemma 3.7(ii). Choose $k$ such that $k\left(n+n_{0}\right) \geq N$. Then by Equation (7)

$$
\# E_{q}(n, k) \leq s_{k\left(n+n_{0}\right)}\left(\delta, B_{k\left(n+n_{0}\right)}\left(\bar{v}^{(n)}, \varepsilon\right)\right)<e^{k\left(n+n_{0}\right) C} .
$$




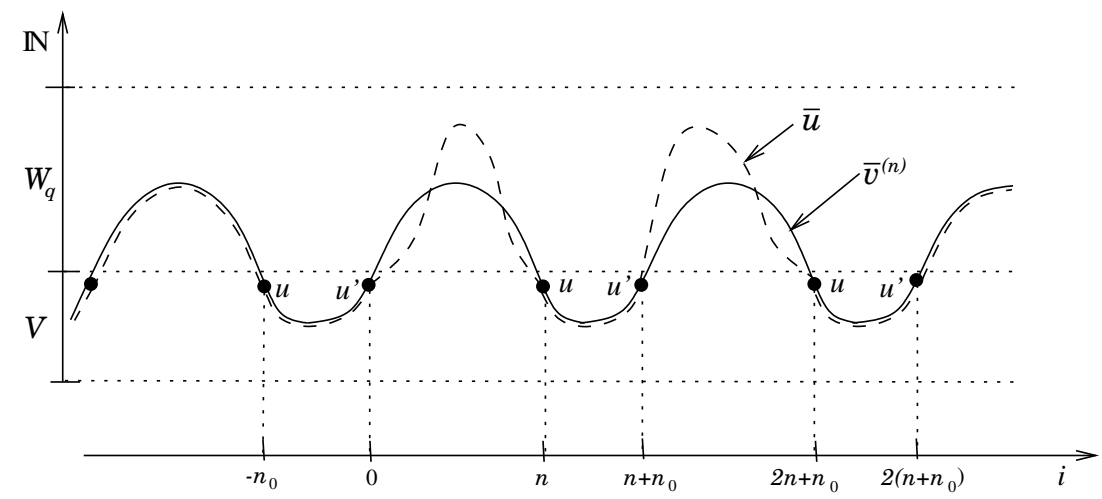

Figure 2. The set $E_{q}(n, k)\left(k=2\right.$ on the picture): $\bar{v}^{(n)}$ (in solid) is a periodic path, $\bar{u}$ (in dashes) is a element of $E_{q}(n, k)$. Between the indices 0 and $k\left(n+n_{0}\right), \bar{v}^{(n)}$ and $\bar{u}$ coincide when $v_{i}^{(n)}$ is in $V$ and $\bar{v}^{(n)}$ and $\bar{u}$ are in $W_{q}$ at the same time. Before 0 or after $k\left(n+n_{0}\right)$, the two paths coincide.

As $\# E_{q}(n, k)=\left(t_{u u^{\prime}}^{W_{q}}(n)\right)^{k}$, one gets $t_{u u^{\prime}}^{W_{q}}(n)<e^{\left(n+n_{0}\right) C}$. This is true for all $q \geq 1$, thus

$$
t_{u u^{\prime}}^{W}(n)=\lim _{q \rightarrow+\infty} t_{u u^{\prime}}^{W_{q}}(n) \leq e^{\left(n+n_{0}\right) C}
$$

and

$$
\tau_{u u^{\prime}}^{W}=\tau_{u v^{\prime}}^{\bar{V}} \leq C<h(V) .
$$

Theorem 3.2 concludes the proof.

Remark 3.9. Define the entropy at infinity as $h_{\infty}(G)=\lim _{n \rightarrow+\infty} h\left(G \backslash G_{n}\right)$ where $\left(G_{n}\right)_{n \geq 0}$ is a sequence of finite graphs such that $\bigcup_{n} G_{n}=G$. The local entropy satisfies $h_{\text {loc }}\left(\Gamma_{G}\right) \geq h_{\infty}(G)$ but in general these two quantities are not equal and the condition $h_{\infty}(G)<h(G)$ does not imply that $G$ is strongly positive recurrent. This is illustrated by Example 2.9 (see Figure 1).

\section{References}

[1] R. Bowen, Entropy-expansive maps, Trans. Amer. Math. Soc., 164 (1972), 323-331, MR 44 \#2907, Zbl 0229.28011.

[2] J. Buzzi, Intrinsic ergodicity of smooth interval maps, Israel J. Math., 100 (1997), 125-161, MR 99g:58071, Zbl 0889.28009.

[3] _ On entropy-expanding maps. Preprint, 2000.

[4] J. Buzzi and S. Ruette, Large topological entropy implies existence of measures of maximal entropy: The case of interval maps. Preprint, 2001. 
[5] M. Denker, C. Grillenberger and K. Sigmund, Ergodic Theory on Compact Spaces, Lecture Notes in Mathematics, 527, Springer-Verlag, 1976, MR 56 \#15879, Zbl 0328.28008.

[6] U. Fiebig, Symbolic Dynamics and Locally Compact Markov Shifts, 1996, Habilitationsschrift, U. Heidelberg.

[7] B.M. Gurevič, Topological entropy of enumerable Markov chains (Russian), Dokl. Akad. Nauk SSSR, 187 (1969), 715-718; English translation: Soviet Math. Dokl., 10(4) (1969), 911-915, MR 41 \#7767, Zbl 0194.49602.

[8] _ Shift entropy and Markov measures in the path space of a denumerable graph (Russian), Dokl. Akad. Nauk SSSR, 192 (1970), 963-965; English translation: Soviet Math. Dokl., 11(3) (1970), 744-747, MR 42 \#3254, Zbl 0217.38101.

[9] B.M. Gurevič and S.V. Savchenko, Thermodynamic formalism for countable symbolic Markov chains (Russian), Uspekhi Mat. Nauk, 53(2) (1998), 3-106; English translation: Russian Math. Surveys, 53(2) (1998), 245-344, MR 2000c:28028, Zbl 0926.37009.

[10] B.M. Gurevič and A.S. Zargaryan, Existence conditions of a maximal measure for a countable symbolic Markov chain (Russian), Vestnik Moskov. Univ. Ser. I Mat. Mekh., 43(5) (1988), 14-18; English translation: Moscow Univ. Math. Bull., 1988, 18-23, MR 91b:58122, Zbl 0717.60083.

[11] S.E. Newhouse, Continuity properties of entropy, Ann. of Math. (2), 129(2) (1989), 215-235; Corrections, 131(2) (1990), 409-410, MR 90f:58108, Zbl 0688.58022, Zbl 0693.58009.

[12] W. Parry, Intrinsic Markov chains, Trans. Amer. Math. Soc., 112 (1964), 55-66, MR 28 \#4579, Zbl 0127.35301.

[13] I.A. Salama, Topological entropy and recurrence of countable chains, Pacific J. Math., 134(2) (1988), 325-341; Errata, 140(2) (1989), 397, MR 90d:54076, MR 90k:54055, Zbl 0619.54031.

[14] _ On the recurrence of countable topological Markov chains, in 'Symbolic dynamics and its applications' (New Haven, CT, 1991), Contemp. Math., 135, 349-360, Amer. Math. Soc., Providence, RI, 1992, MR 93m:54071, Zbl 0801.54032.

[15] O.M. Sarig, Phase transitions for countable Markov shifts, Comm. Math. Phys., 217(3) (2001), 555-577, MR 2002b:37040.

[16] D. Vere-Jones, Geometric ergodicity in denumerable Markov chains, Quart. J. Math. Oxford Ser. (2), 13 (1962), 7-28, MR 25 \#4571, Zbl 0104.11805.

Received April 26, 2001 and revised June 20, 2002.

Institut DE MAThÉmATiQUes DE LUMiny

CNRS UPR 9016

163 AVENUE DE LUMiny, CASE 907

13288 MARSEILLE CEDEX 9

FRANCE

E-mail address: ruette@iml.univ-mrs.fr 